\title{
Mutation analysis of a Chinese pedigree with triphalangeal thumb-polysyndactyly syndrome
}

\author{
X.S. Xing ${ }^{1}$, H.W. Zhu' ${ }^{2}$, C. Chen ${ }^{1}$, S.S. Wang ${ }^{1}$, Y. Luo ${ }^{1}$ and X. Zhang ${ }^{2}$ \\ ${ }^{1}$ Research Center for Medical Genomics, Key Laboratory of Cell Biology, \\ Ministry of Public Health, Key Laboratory of Medical Cell Biology, \\ Ministry of Education, China Medical University, Shenyang, China \\ ${ }^{2}$ McKusick-Zhang Center for Genetic Medicine and State Key Laboratory \\ of Medical Molecular Biology, Institute of Basic Medical Sciences, \\ Chinese Academy of Medical Sciences \& Peking Union Medical College, \\ Beijing, China
}

Corresponding authors: Y. Luo / X. Zhang

E-mail: luoyang@mail.cmu.edu.cn / xuezhang@pumc.edu.cn

Genet. Mol. Res. 13 (1): 246-254 (2014)

Received November 21, 2012

Accepted April 11, 2013

Published January 17, 2014

DOI http://dx.doi.org/10.4238/2014.January.17.8

\begin{abstract}
Triphalangeal thumb-polysyndactyly syndrome (TPTPS) is an autosomal dominant limb disorder with triphalangeal thumbs, polysyndactyly, and syndactyly. In this study, we describe a fourgeneration Han Chinese family with eight affected members. Haplotype analysis, Affymetrix SNP 6.0 arrays, qPCR, and gap-PCR were performed. Haplotyping results linked the disease-causing region to the $7 \mathrm{q} 36$ region that includes the zone of polarizing activity-regulatory sequence. A 442-kb duplication was found on chromosome 7 that cosegregated with the disease phenotype. The extent of the duplication was determined by qPCR, and the breakpoints were identified by gapPCR and direct sequencing. This mutation was not detected in normal members in the same family. Our data therefore suggest that this novel microduplication, between $155,913,768$ and $156,355,553$ bp on chromosome 7, could be considered the cause of TPTPS in this kindred.
\end{abstract}

Key words: TPTPS; Zone of polarizing activity-regulatory sequence; Microduplication 


\section{INTRODUCTION}

Triphalangeal thumb-polysyndactyly syndrome (TPTPS; OMIM 174,500) is an autosomal dominant disorder with complete penetrance and variable expression. Typical phenotypes are triphalangeal thumbs, syndactylous fingers 3-5 or milder syndactyly of fingers 4 and 5 . Finger 2 is always normal and the feet are usually less severely affected. TPTPS was first reported in a large Dutch family by Nicolai and Hamel in 1988. The disease locus was mapped to chromosome 7q36, which is also the location of preaxial polydactyly type 2 or 3 (Tsukurov et al., 1994; Balci et al., 1999; Wang et al., 2007). Point mutations in a noncoding region, intron 5 of the LMBR1 gene, were shown to result in TPTPS (Lettice et al., 2003). In addition to point mutations, duplications that involve the zone of polarizing activity (ZPA)-regulatory sequence (ZRS), also cause TPTPS (Klopocki et al., 2008; Sun et al., 2008). Further studies showed that mutations in the murine region homologous to ZRS caused hemimelic extra toes, Sasquatch, M101116, and M100081 (Lettice et al., 2002, 2003; Masuya et al., 2007). Mutations in ZRS have also been shown to result in supernumerary digits on chick limbs (Maas and Fallon, 2004). ZRS sequences are highly conserved from mammals to fish and have been identified as long-range limb-specific enhancers for the sonic hedgehog $(\mathrm{SHH})$ gene (Lettice et al., 2002, 2003; Sagai et al., 2004). ZRS is located about $1 \mathrm{Mb}$ upstream of the target gene. The SHH protein is a key regulator in defining limb anterior-posterior axes in early embryogenesis (Lettice and Hill, 2005; Hill, 2007). Here, we found that a microduplication in 7q36 containing the ZRS region is the pathogenic mutation underlying TPTPS in a large Chinese family.

\section{MATERIAL AND METHODS}

\section{Subjects}

In this study, we investigated a four-generation Han Chinese family consisting of 23 individuals. After physical examination, digital photographs were taken to document limb phenotypes in affected individuals. Blood samples were collected from 10 family members (III-1, III-2, III-5, III-7, III-8, III-12, III-13, IV-1, IV-5, IV-7) with informed consent and approval of the China Medical University Institutional Review Board.

\section{Haplotype analysis}

Genomic DNA was extracted from venous blood samples using a Universal Genomic DNA Extraction Kit Ver. 3.0 (TaKaRa, China). Haplotype analysis was performed with seven microsatellite markers in the region close to $\mathrm{SHH}$ and the ZRS in 7q36, as previously described (Sun et al., 2008).

\section{Affymetrix SNP 6.0 arrays and copy number analysis}

To test whether TPTPS was caused by a copy number variant (CNV), we performed a genome-wide high-resolution $\mathrm{CNV}$ scan in the proband. Blood samples were sent to the CapitalBio Corporation (Beijing, China) where genomic DNA was extracted, dissolved in Tris-HCl, $\mathrm{pH}$ 8.0, and hybridized according to the manufacturer protocol. CNV identification was performed with the Affymetrix Genotyping Console 3.0 software. 


\section{Real-time quantitative PCR}

To confirm the result of the arrays and determine the extent of the duplication, two subsets of primers were designed. The first subset was located in the duplicated region identified in the array analysis. The other primers were evenly distributed over the sequences flanking the duplicated region (mean distance, $2 \mathrm{~kb}$ ). Primer sequences can be obtained upon request.

qPCR was performed in a total volume of $25 \mu \mathrm{L}$. Each tube contained $12.5 \mu \mathrm{L}$ SYBR Premix Ex Taq (TaKaRa, China), 50 ng genomic DNA $(5 \mu \mathrm{L})$, and 4 x $2.5 \mu \mathrm{L}$ primers ( $250 \mathrm{nM}$ each). Reactions were carried out using the Rotor-Gene 2000 Real-Time Cycler (Applied Biosystems, USA). The qPCR condition consisted of an initial denaturation step of $95^{\circ} \mathrm{C}$ for $20 \mathrm{~s}$, followed by 40 cycles of amplification at $95^{\circ} \mathrm{C}$ for $30 \mathrm{~s}, 95^{\circ} \mathrm{C}$ for $5 \mathrm{~s}$, and $60^{\circ} \mathrm{C}$ for $20 \mathrm{~s}$.

Samples were run in triplicate in separate tubes, and the average of $\Delta \Delta \mathrm{Ct}$ values of different samples was analyzed to calculate the relative copy number $(\mathrm{RCN})$, using a fragment on chromosome 21 as control (Lyle et al., 2006).

\section{Gap-PCR}

Gap-PCR was used to obtain breakpoint information. Primers were the same as for the qPCRs mentioned above. PCR was performed as follows: 2X GC PCR buffer I, $125 \mathrm{U}$ (5 U/ $\mu \mathrm{L}$ ) LA Taq DNA polymerase (TaKaRa, China), $250 \mathrm{nmol}$ dNTPs, $10 \mu \mathrm{mol}$ of each primer, and approximately $100 \mathrm{ng}$ template DNA in a total volume of $50 \mu \mathrm{L}$. PCR was $5 \mathrm{~min}$ at $94^{\circ} \mathrm{C}$, followed by 35 cycles at $94^{\circ} \mathrm{C}$ for $30 \mathrm{~s}, 60^{\circ} \mathrm{C}$ for $30 \mathrm{~s}$, and $72^{\circ} \mathrm{C}$ for $2 \mathrm{~min}$. Amplified products were directly sequenced using the same primers as for PCR.

\section{RESULTS}

\section{Clinical characteristics}

The family consisted of 23 individuals with eight members affected by TPTPS. Phenotypes varied among affected individuals, but none exhibited foot abnormalities. Examples of hand phenotypes are shown in Figure 1. Among the three affected female members, one displayed triplicated thumbs and syndactyly of fingers 3-5 (Figure 1A), one simply showed the typical TPTPS hand phenotype (Figure 1C), and all had normal index fingers. The affected males in this family exhibited a severe phenotype, with complete syndactyly between the duplicated thumbs and index fingers, and with complete syndactyly of fingers 3-5 (Figure 1B). Basic hand form and function could be restored with surgery (Figure 1D).

\section{Haplotype analysis}

Haplotype analysis after sequence-tagged-site marker analysis showed that all affected individuals share the same haplotype: 1-2-5-3-3-3, while unaffected members display a different haplotype (Figure 2). This confirmed the TPTPS locus as the $7 \mathrm{q} 36$ region that includes ZRS. 

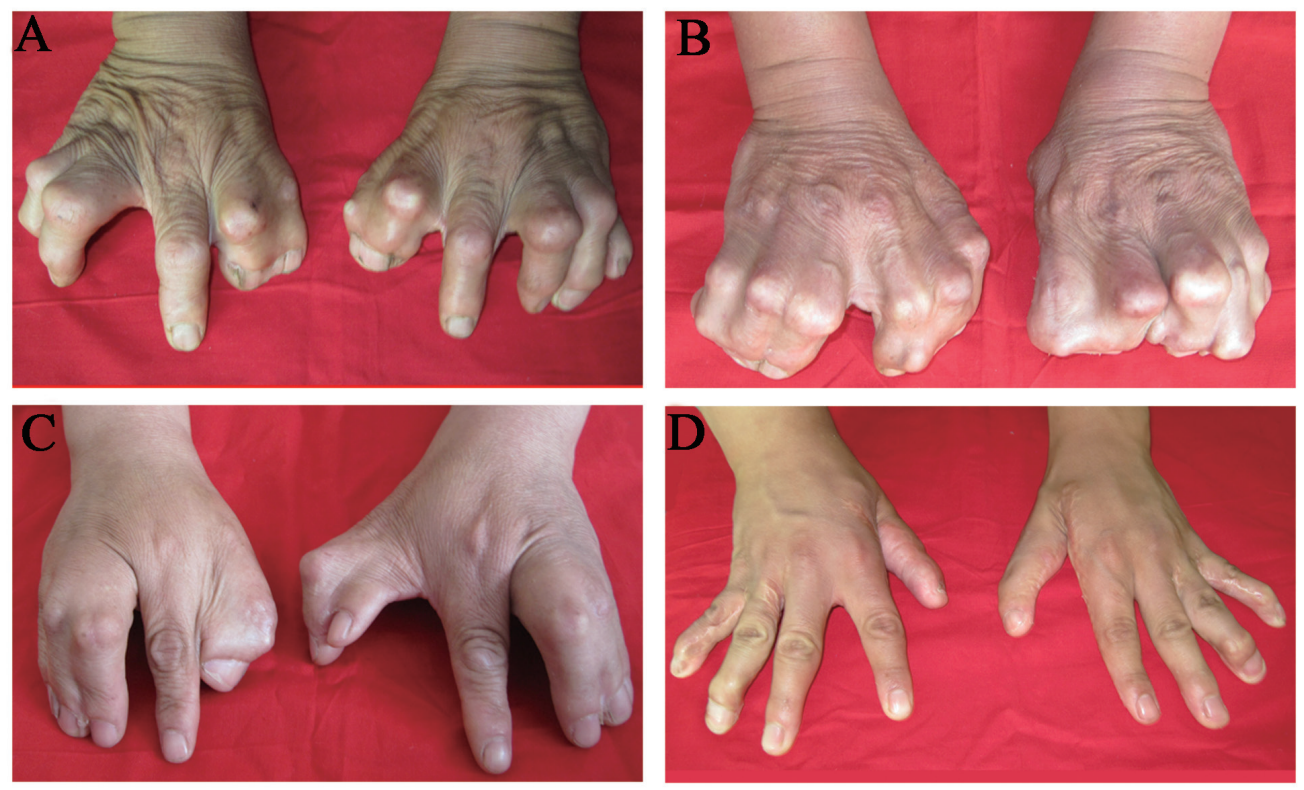

Figure 1. Photographs from individuals with TPTPS, III-2 (A), III-7 (B), III-12 (C), IV-7 after operations (D).

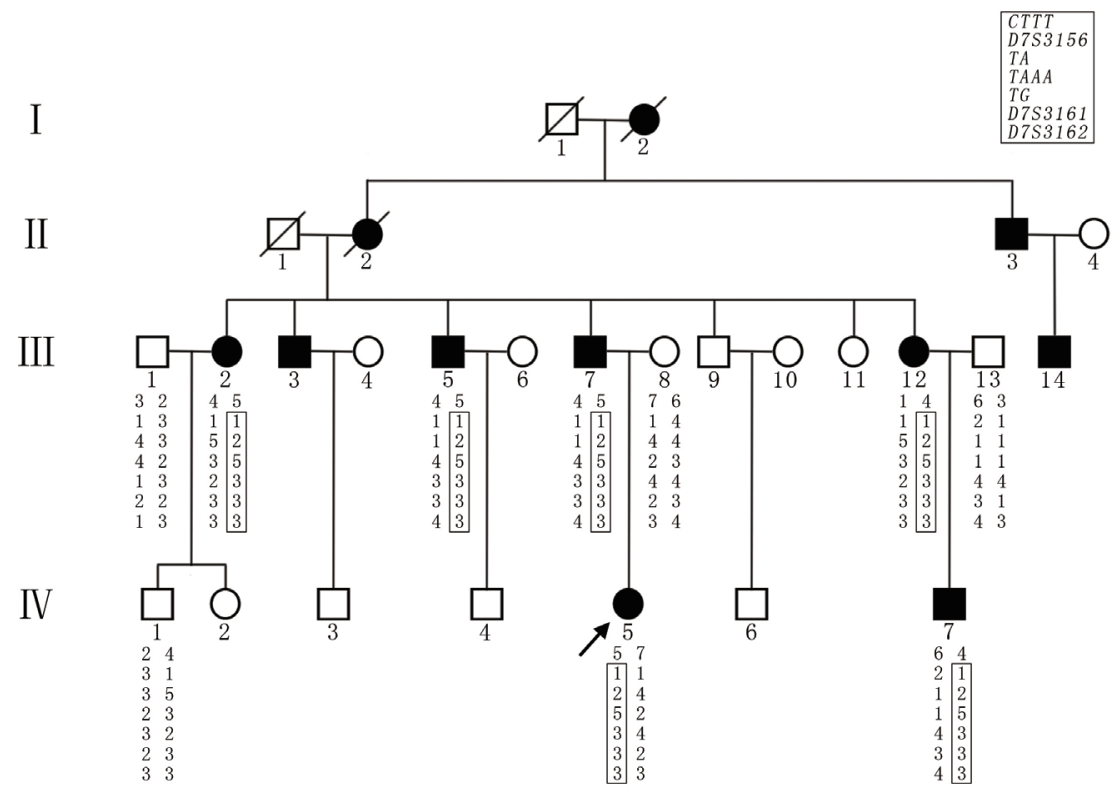

Figure 2. Pedigrees and disease-haplotype segregation of the Han Chinese family with TPTPS. Filled symbols represent affected individuals with TPTPS, and open symbols represent individuals without TPTPS. Squares and circles indicate males and females, respectively. The arrow identifies the proband. The solid bar indicates the critical region. TPTPS $=$ triphalangeal thumb-polysyndactyly syndrome. 


\section{Identification of the ZRS duplication}

In the proband, using the Affymetrix SNP 6.0 arrays, a $>400-\mathrm{kb}$ microduplication was found on 7q36.3 that included the ZRS (CN_1,202,035 to SNP_A-2,207,244). We then performed qPCR to examine the $\mathrm{RCN}$ of the $\mathrm{ZRS}$ region in all samples from the family. An $\mathrm{RCN}$ of 1.58 (mean value $=1.42-1.70$ ) in affected individuals was detected; no copy number mutations were detected in normal individuals, confirming that the duplication co-segregated with the TPTPS phenotype in this family (Figure 3A). A value of 1.5 for the RCN was taken to indicate a duplication.

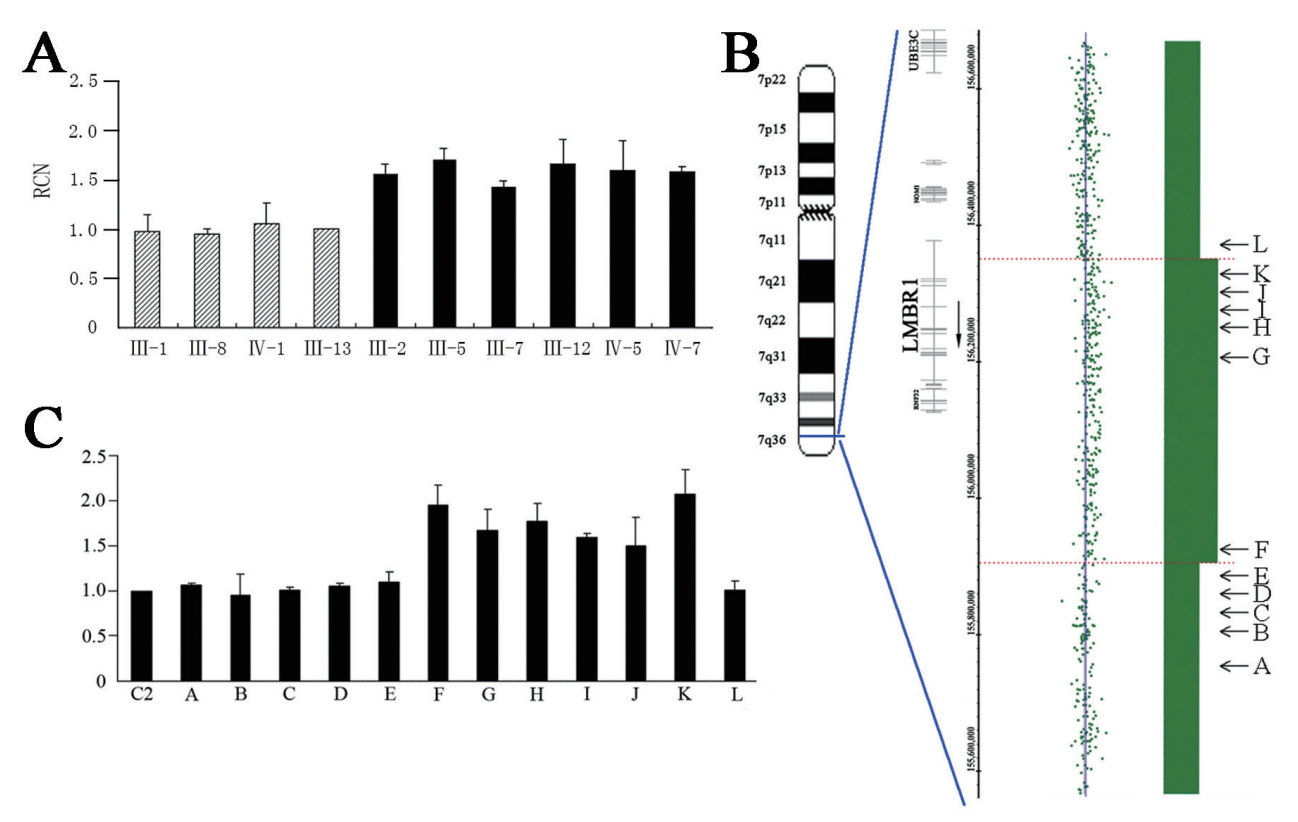

Figure 3. Detection of duplications. A. Quantitative polymerase chain reaction (qPCR) assay confirming the ZRS duplication in the family members. The $2^{-\Delta \Delta \mathrm{Ct}}$ ratios are reported in the $\mathrm{y}$-axis for 4 unaffected individuals (hatched bars) and 6 affected individuals (black bars). RCN $=$ relative copy number. Error bars represent SD. B. Copynumber state of a 400-kb genomic region on chromosome 7q36 in the proband. Positions of the genes and qPCR amplications (horizontal arrows A to L) are indicated. C. Determination of the duplication extent by qPCR assay. $\mathrm{C} 2$ represents the control gene.

\section{Refining the extent of duplication and sequencing the breakpoint}

By 12 additional qPCR analyses, we localized the breakpoint to a region of about 2.2 $\mathrm{kb}$ between primers $\mathrm{E}$ and $\mathrm{F}$, and $1.9 \mathrm{~kb}$ between primers $\mathrm{K}$ and $\mathrm{L}$ (Figure $3 \mathrm{C}$ ).

By PCR amplification on genomic DNA with primers K-forward and E-reverse, a nearly 2000-bp fragment containing the transition site between the telomeric and centromeric breakpoints was detected in affected individuals and was absent in nonaffected individuals (Figure 4A and B). We identified the breakpoints by direct sequencing (Figure 4C). The centromeric breakpoint of the microduplication was between nucleotide $155,913,767$ and 
$155,913,768 \mathrm{bp}$, and the telomeric was between nucleotide $156,355,553$ and $156,355,554$ bp on chromosome 7 (positions according to UCSC, March 2006). The exact length of the duplicated fragment was confirmed to be $441,786 \mathrm{bp}$.
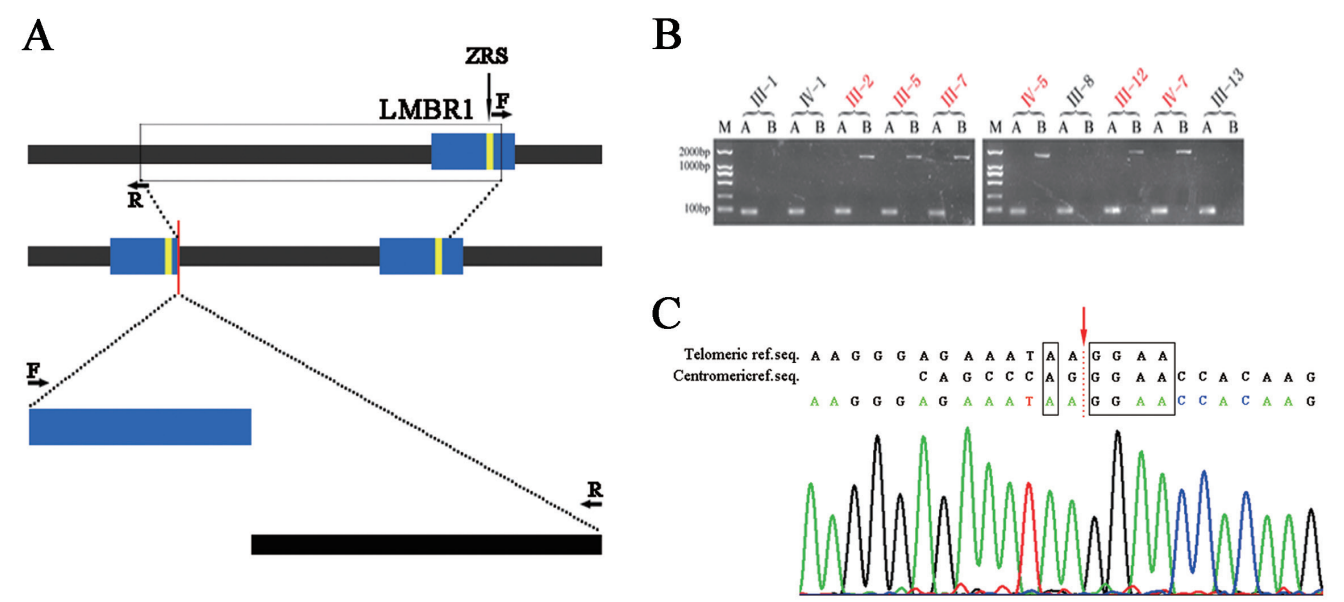

Figure 4. Identification of the breakpoints. A. Schematic diagram showing the possible molecular mechanism of the duplication. $\mathrm{F}=$ forward primer; $\mathrm{R}=$ reverse primer. B. PCR amplification of the junction. A fragment containing the breakpoints was amplified in all the family members (red = affected individuals; black $=$ unaffected individuals). C. Breakpoint identification by sequence analysis. The top line displays the telomeric reference sequence, the second line displays the centromeric reference sequence and the third line shows result of sequence analysis. The vertical arrow shows the breakpoints. The microhomologies are round by the black squares.

\section{DISCUSSION}

In this study, we investigated a Han Chinese family with typical TPTPS. Haplotype analysis indicated that the region responsible was linked to the ZRS region on chromosome 7q36.3. We found a genomic microduplication involving the ZRS long-range enhancer in this TPTPS family. The duplication co-segregated with the disease phenotype and was not present in unaffected family members. This result strongly suggests that the duplication involving ZRS is the pathogenic mutation causing TPTPS in this family. ZPA is important in limb morphogenesis and development (Hill, 2007). The SHH protein, which is produced and secreted by mesenchymal cells, determines the type and number of digits within the ZPA. ZRS is a cis-regulatory element of SHH that can activate a reporter gene expressed in the ZPA (Lettice et al., 2003; Sagai, et al., 2004), and is necessary for proper posterior expression in limb development (Sagai et al., 2005). Subsequent research on human patients with isolated limb malformations revealed many mutations affecting the ZRS region (VanderMeer and Ahituv, 2011). According to the reports, both single-base pair substitutions and duplications that encompass the ZRS region can be found in TPTPS patients (Wang et al., 2007).

Including the results of our study, ZRS duplications of eleven different sizes have been detected to date. The largest duplicated region, which included all of the other duplicated regions, was approximately $588 \mathrm{~kb}$ (Figure 5), for which the resultant phenotype was a small extra-phalange between the proximal and distal phalanges, which is the minimal variation in a 
triphalangeal thumb (Klopocki et al., 2008). In contrast, the minimal duplication appears to be nearly $73 \mathrm{~kb}$, but causes complete cutaneous syndactyly of all fingers and polydactyly (Wieczorek et al., 2010). Besides those due to duplications, the phenotypes of different point mutations in the ZRS are not the same. In addition to TPTPS, ZRS mutations can cause other phenotypes. Wieczorek reported a family with syndactyly type IV (SD4; OMIM186200). In the same report, a point mutation of ZRS caused Werner mesomelic syndrome, which is also called tibial hemimelia-polysyndactyly-triphalangeal thumb with fibular dimelia (OMIM188770). Moreover, a family consisting of a father with TPTPS and a daughter with tibial hemimeliapolysyndactyly-triphalangeal thumb syndrome was reported (Kantaputra and Chalidapong, 2000). Numerous TPTPS families have no detected mutation or duplication in ZRS, but the symptoms are strongly that linked to the ZRS (Lettice et al., 2003; Gurnett et al., 2007; Li et al., 2009). These findings suggest there is no correlation between phenotypic severity and the extent of duplication or the type of mutation. TPTPS may be an intermediate phenotype in these complex polysyndactyly syndromes. Reviewing the duplications of the ZRS region, we found that they all cover the region from 156265512 to 156281894 bp on chromosome 7. Sun et al. (2008) suggested that the smallest region of overlap between the various sizes of duplication in different families might contain the critical disease region. Therefore, we propose that the other regions maybe include other regulatory factors that produce the various phenotypes. In addition, a duplication that contains intact regulatory factors might have less effect than those where the breakpoints disrupt the integrity of regulatory factors. This may be because an activating or repressing protein normally binds to the ZRS and modulates the regulation for a gain-of-function effect. This could be the reason for this disease (Klopocki et al., 2008).

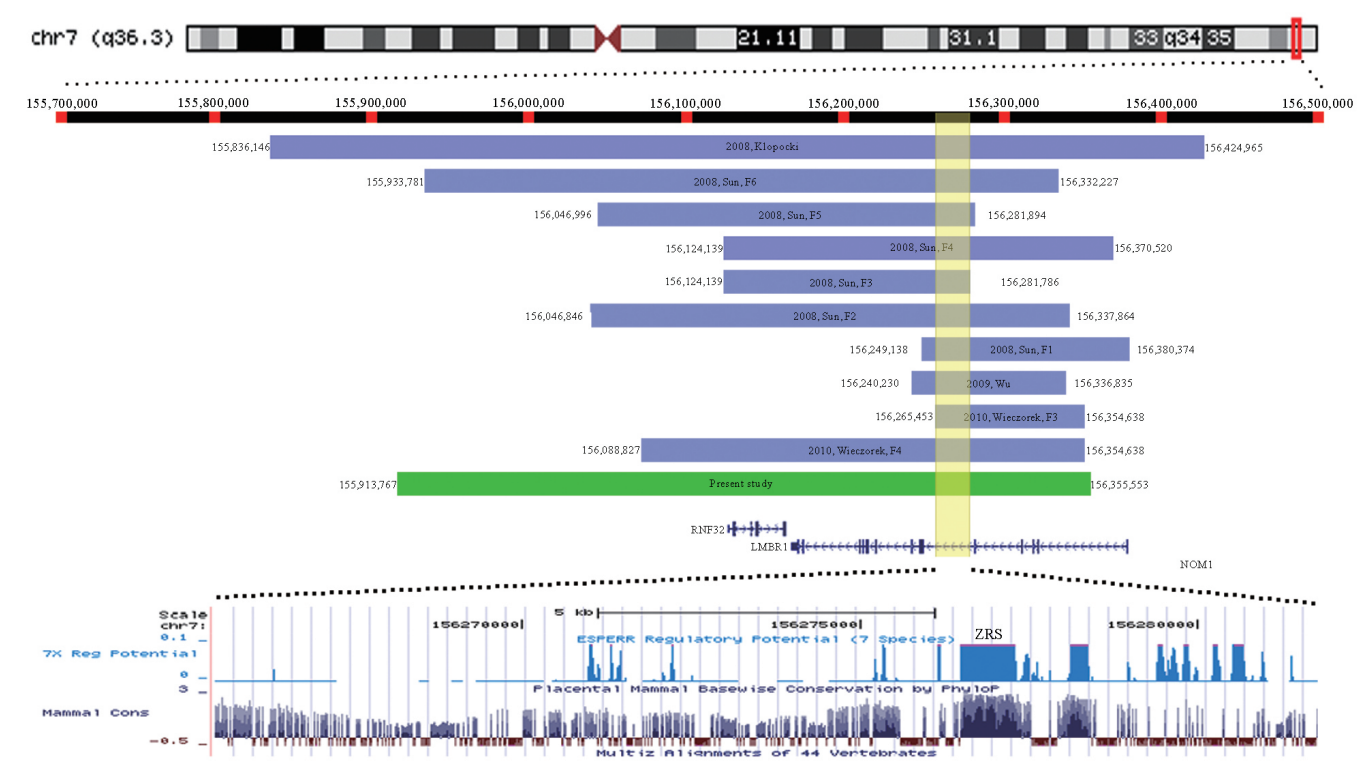

Figure 5. Genomic context of ZRS duplications. Each bar in the track indicates the region that is duplicated in a family with TPTPS. The blue ones were reported previously, while the green one was the present study. The yellow is a region included in each bar. The part below was the location, conservation and the regulatory potential of the yellow region. Images generated using the UCSC genome browser, http://genome.ucsc.edu/index.html. 
So far, many disease-associated CNVs have been reported (Lupski and Stankiewicz, 2005; Lee and Lupski, 2006). These genomic disorders are usually caused by structural changes resulting from genomic instability, or arise because of susceptible genome architecture (Lupski, 1998, 2009). The literature shows that chromosome 7 is a segmental, duplication-enriched chromosome, where $30 \%$ of the complex chromosomal rearrangements have breakpoints (Vermeulen et al., 2004). Klopocki and Ott identified a non-homologous recombination in a TPTPS family that is mediated by topoisomerases (see Klopocki et al., 2008). We investigated the exact breakpoint of the TPTPS family that we studied using sequence analysis. Unlike his report, the genomic structural change in our case was not mediated by topoisomerases. The telomeric breakpoint was in the middle of a long interspersed nuclear element, LIMB4. Beside the breakpoint microhomologies of 1-5 nucleotides, no other significant sequence homologies were found. In addition, TTTAAA, a curved DNA signal, and AT-rich signals were observed at the breakpoint junctions. These findings suggest that this conformation might form DNA hairpins during replication that could interact with the nuclear matrix (Singh et al., 1997). Nonhomologous end-joining (NHEJ) is the major cause of genomic duplications; it happens throughout the cell cycle, with no strict requirement for microhomologous sequences, four or fewer nucleotides being sufficient. In light of the features of breakpoints identified in the family in this study, we propose NHEJ as the mechanism for the rearrangement we observed.

Duplication is a major type of copy number mutation in many human genetic diseases (Beckmann et al., 2007). In this study, we combined Affymetrix SNP array 6.0 and qPCR for fine mapping of the duplicated DNA region. The array contains more than 906,600 SNPs and 946,000 copy-number probes that cover the general region of duplication or deletion. qPCR assay was then used to define the duplicated region more closely. Since neither technique could identify breakpoints, gap-PCR and direct sequencing were necessary to provide insights into the possible mechanism.

In conclusion, we identified a 441,786-bp genomic duplication containing ZRS regions as a limb-specific $\mathrm{SHH}$ enhancer, and analyzed the possible mechanism causing the rearrangement. More investigations are needed to clarify the role of ZRS in the pathogenesis of the complex disease, TPTPS.

\section{ACKNOWLEDGMENTS}

We thank all the family members for their generous participation. Research supported by the National Natural Science Foundation of China (\#81170543), the National Basic Research Program of China (\#2012CB944600), and the Specialized Research Fund for the Doctoral Program of Higher Education of China (\#20102104120024).

\section{REFERENCES}

Balci S, Demirtas M, Civelek B, Piskin M, et al. (1999). Phenotypic variability of triphalangeal thumb-polysyndactyly syndrome linked to chromosome 7q36. Am. J. Med. Genet. 87: 399-406.

Beckmann JS, Estivill X and Antonarakis SE (2007). Copy number variants and genetic traits: closer to the resolution of phenotypic to genotypic variability. Nat. Rev. Genet. 8: 639-646.

Gurnett CA, Bowcock AM, Dietz FR, Morcuende JA, et al. (2007). Two novel point mutations in the long-range SHH enhancer in three families with triphalangeal thumb and preaxial polydactyly. Am. J. Med. Genet. A 143: 27-32.

Hill RE (2007). How to make a zone of polarizing activity: insights into limb development via the abnormality preaxial polydactyly. Dev. Growth Differ. 49: 439-448. 
Kantaputra PN and Chalidapong P (2000). Are triphalangeal thumb-polysyndactyly syndrome (TPTPS) and tibial hemimelia-polysyndactyly-triphalangeal thumb syndrome (THPTTS) identical? A father with TPTPS and his daughter with THPTTS in a Thai family. Am. J. Med. Genet. 93: 126-131.

Klopocki E, Ott CE, Benatar N, Ullmann R, et al. (2008). A microduplication of the long range SHH limb regulator (ZRS) is associated with triphalangeal thumb-polysyndactyly syndrome. J. Med. Genet. 45: 370-375.

Lee JA and Lupski JR (2006). Genomic rearrangements and gene copy-number alterations as a cause of nervous system disorders. Neuron 52: 103-121.

Lettice LA and Hill RE (2005). Preaxial polydactyly: a model for defective long-range regulation in congenital abnormalities. Curr. Opin. Genet. Dev. 15: 294-300.

Lettice LA, Horikoshi T, Heaney SJ, van Baren MJ, et al. (2002). Disruption of a long-range cis-acting regulator for Shh causes preaxial polydactyly. Proc. Natl. Acad. Sci. U. S. A. 99: 7548-7553.

Lettice LA, Heaney SJ, Purdie LA, Li L, et al. (2003). A long-range Shh enhancer regulates expression in the developing limb and fin and is associated with preaxial polydactyly. Hum. Mol. Genet. 12: 1725-1735.

Li H, Wang CY, Wang JX, Wu GS, et al. (2009). Mutation analysis of a large Chinese pedigree with congenital preaxial polydactyly. Eur. J. Hum. Genet. 17: 604-610.

Lupski JR (1998). Genomic disorders: structural features of the genome can lead to DNA rearrangements and human disease traits. Trends Genet. 14: 417-422.

Lupski JR (2009). Genomic disorders ten years on. Genome Med. 1: 42.

Lupski JR and Stankiewicz P (2005). Genomic disorders: molecular mechanisms for rearrangements and conveyed phenotypes. PLoS Genet. 1: e49.

Lyle R, Radhakrishna U, Blouin JL, Gagos S, et al. (2006). Split-hand/split-foot malformation 3 (SHFM3) at 10q24, development of rapid diagnostic methods and gene expression from the region. Am. J. Med. Genet. A 140: 1384-1395.

Maas SA and Fallon JF (2004). Isolation of the chicken Lmbr1 coding sequence and characterization of its role during chick limb development. Dev. Dyn. 229: 520-528.

Masuya H, Sezutsu H, Sakuraba Y, Sagai T, et al. (2007). A series of ENU-induced single-base substitutions in a longrange cis-element altering Sonic hedgehog expression in the developing mouse limb bud. Genomics 89: 207-214.

Nicolai JP and Hamel BC (1988). A family with complex bilateral polysyndacty. J. Hand Surg. Am. 13: 405-407.

Sagai T, Masuya H, Tamura M, Shimizu K, et al. (2004). Phylogenetic conservation of a limb-specific, cis-acting regulator of Sonic hedgehog (Shh). Mamm. Genome 15: 23-34.

Sagai T, Hosoya M, Mizushina Y, Tamura M, et al. (2005). Elimination of a long-range cis-regulatory module causes complete loss of limb-specific Shh expression and truncation of the mouse limb. Development 132: 797-803.

Singh GB, Kramer JA and Krawetz SA (1997). Mathematical model to predict regions of chromatin attachment to the nuclear matrix. Nucleic Acids Res. 25: 1419-1425.

Sun M, Ma F, Zeng X, Liu Q, et al. (2008). Triphalangeal thumb-polysyndactyly syndrome and syndactyly type IV are caused by genomic duplications involving the long range, limb-specific SHH enhancer. J. Med. Genet. 45: 589-595.

Tsukurov O, Boehmer A, Flynn J, Nicolai JP, et al. (1994). A complex bilateral polysyndactyly disease locus maps to chromosome 7q36. Nat. Genet. 6: 282-286.

VanderMeer JE and Ahituv N (2011). Cis-regulatory mutations are a genetic cause of human limb malformations. Dev. Dyn. 240: 920-930.

Vermeulen S, Menten B, Van Roy N, Van Limbergen H, et al. (2004). Molecular cytogenetic analysis of complex chromosomal rearrangements in patients with mental retardation and congenital malformations: delineation of 7q21.11 breakpoints. Am. J. Med. Genet. A 124A: 10-18.

Wang ZQ, Tian SH, Shi YZ, Zhou PT, et al. (2007). A single C to T transition in intron 5 of LMBR1 gene is associated with triphalangeal thumb-polysyndactyly syndrome in a Chinese family. Biochem. Biophys. Res. Commun. 355 : 312-317.

Wieczorek D, Pawlik B, Li Y, Akarsu NA, et al. (2010). A specific mutation in the distant sonic hedgehog (SHH) cisregulator (ZRS) causes Werner mesomelic syndrome (WMS) while complete ZRS duplications underlie Haas type polysyndactyly and preaxial polydactyly (PPD) with or without triphalangeal thumb. Hum. Mutat. 31: 81-89. 